OPEN ACCESS

Edited by:

Ata Murat Kaynar,

University of Pittsburgh, United States

Reviewed by:

Longxiang Su,

Peking Union Medical College

Hospital (CAMS), China

Yihua Dong,

Wenzhou Medical University, China

${ }^{*}$ Correspondence:

Shih-Chi Ku

scku1015@ntu.edu.tw

tThese authors have contributed equally to this work

Specialty section:

This article was submitted to Intensive Care Medicine and

Anesthesiology,

a section of the journal

Frontiers in Medicine

Received: 05 January 2021 Accepted: 19 April 2021

Published: 07 June 2021

Citation:

Huang TT-M, Chien $Y-C$, Wang $C-H$, Chang S-Y, Wang J-T, Hsieh S-C,

Yeh Y-C, Ku S-C, Yu C-J, Chiang B-L,

Chang S-C and To/wani A (2021)

Successful Treatment of a Critically III

COVID-19 Patient Using Continuous

Renal Replacement Therapy With

Enhanced Cytokine Removal and

Tocilizumab: A Case Report.

Front. Med. 8:649583.

doi: 10.3389/fmed.2021.649583

\section{Successful Treatment of a Critically III COVID-19 Patient Using Continuous Renal Replacement Therapy With Enhanced Cytokine Removal and Tocilizumab: A Case Report}

\author{
Thomas Tao-Min Huang ${ }^{1 \dagger}$, Ying-Chun Chien ${ }^{1 \dagger}$, Chih-Hsien Wang ${ }^{2}$, Sui-Yuan Chang ${ }^{3}$, \\ Jann-Tay Wang ${ }^{1}$, Song-Chou Hsieh ${ }^{1}$, Yu-Chang Yeh ${ }^{4}$, Shih-Chi Ku ${ }^{1 *}$, Chong-Jen Yu ${ }^{1}$, \\ Bor-Luen Chiang ${ }^{5}$, Shan-Chwen Chang ${ }^{1}$ and Ashita Tolwani ${ }^{6}$ \\ ${ }^{1}$ Department of Internal Medicine, National Taiwan University Hospital and College of Medicine, Taipei, Taiwan, ${ }^{2}$ Department \\ of Surgery, National Taiwan University Hospital and College of Medicine, Taipei, Taiwan, ${ }^{3}$ Department of Clinical Laboratory \\ Sciences and Medical Biotechnology, National Taiwan University College of Medicine, Taipei, Taiwan, ${ }^{4}$ Department of \\ Anesthesiology, National Taiwan University Hospital and College of Medicine, Taipei, Taiwan, ${ }^{5}$ Department of Pediatrics, \\ National Taiwan University Hospital and College of Medicine, National Taiwan University, Taipei, Taiwan, ${ }^{6}$ Division of \\ Nephrology, University of Alabama, Birmingham, AL, United States
}

The COVID-19 pandemic has caused multiple deaths worldwide. Since no specific therapies are currently available, treatment for critically ill patients with COVID-19 is supportive. The most severe patients need sustained life support for recovery. We herein describe the course of a critically ill COVID-19 patient with multi-organ failure, including acute respiratory failure, acute kidney injury, and fulminant cytokine release syndrome (CRS), who required mechanical ventilation and extracorporeal membrane oxygenation support. This patient with a predicted high mortality risk was successfully managed with a careful strategy of oxygenation, uremic toxin removal, hemodynamic support, and most importantly, cytokine-targeted intervention for CRS, including cytokine/endotoxin removal, anti-cytokine therapy, and immune modulation. Comprehensive cytokine data, CRS parameters, and biochemical data of extracorporeal removal were provided to strengthen the rationale of this strategy. In this report, we demonstrate that timely combined hemoperfusion with cytokine adsorptive capacity and anti-cytokine therapy can successfully treat COVID-19 patients with fulminant CRS. It also highlights the importance of implementing cytokine-targeted therapy for severe COVID-19 guided by the precise measurement of disease activity.

\footnotetext{
Keywords: COVID-19, cytokine release syndrome, extracorporeal membrane oxygenation, continuous renal replacement therapy, tocilizumab
}

\section{INTRODUCTION}

The emerging and rapid transmission of a novel coronavirus, severe acute respiratory syndrome coronavirus 2 (SARS-CoV-2), with its associated syndrome, coronavirus disease (COVID-19), has spread worldwide after its outbreak in Wuhan, China in late 2019 (1). Although most patients may be asymptomatic, patients with advanced ages or multiple comorbidities can develop 
fatal illnesses and rapidly succumb to them. It is estimated that $2-10 \%$ of the patients may develop critical illness and may need intensive care and advanced life support (1-5). The associated pathophysiology is diverse and might range from transient organ dysfunction to deadly multi-organ failure. The organs involved include the cardiovascular system, kidneys, lungs, hematologic system, and immune system. Severely ill patients may have cytokine release syndrome (CRS) (6), presenting with refractory hypotension, hemophagocytic lymphohistiocytosis (7), antiphospholipid activity (8), and kidney damage (9). Since effective antiviral therapy is not available at this time; life-supportive measures and effective complication management are pivotal measures for patient survival. We report herein a successful treatment strategy using cytokine-targeted therapy, including CRS management and extracorporeal cytokine removal, in a critically ill COVID-19 patient with a devastating clinical course.

\section{CASE DESCRIPTION}

A 53-year-old man with a history of colon cancer and bladder cancer with disease-free status for more than 5 years developed watery diarrhea $3 \mathrm{~d}$ after close contact with a confirmed COVID19 patient. Fever (body temperature, $37.7^{\circ} \mathrm{C}$ ), general malaise, myalgia, and poor appetite prompted him to seek medical advice on the 11th day, and he was admitted to a regional hospital. The hemogram was normal (white blood cell count: $4,900 / \mu \mathrm{L}$, hemoglobin concentration: $16.6 \mathrm{~g} / \mathrm{dL}$, and platelet count: 203 $\mathrm{k} / \mu \mathrm{L}$ ). The aspartate aminotransferase (AST) and alanine aminotransferase (ALT) levels were 44 and $45 \mathrm{U} / \mathrm{L}$, respectively. There was an elevation in the C-reactive protein (CRP) level $(12.5 \mathrm{mg} / \mathrm{dL})$, although the procalcitonin level was within normal limits $(0.15 \mathrm{ng} / \mathrm{mL})$. Polymerase chain reaction (PCR) from a nasal swab for SARS-CoV-2 was positive. Moxifloxacin (400 mg daily) and hydroxychloroquine were administered.

On day 16, he developed respiratory distress with an escalation of his oxygen needs from nasal prongs to a nonrebreathing mask. A chest radiograph revealed rapid progression of pulmonary infiltrates (Figure 2A). He underwent tracheal intubation for severe hypoxemia with an arterial oxygen partial pressure to fractional inspired oxygen ratio of $109 \mathrm{mmHg}$. In addition, he had lactic acidosis (lactic acid level $2.3 \mathrm{mg} / \mathrm{dL}$ ), rhabdomyolysis (creatinine kinase level $261 \mathrm{U} / \mathrm{L}$ ), high CRP level (14.6 mg/dL), and hyperferritinemia (ferritin level 2,957 ng/mL). His urine volume was $\sim 840 \mathrm{~mL}$ in $24 \mathrm{~h}$, and the renal reserve was adequate with a serum creatinine level of $1.1 \mathrm{mg} / \mathrm{dL}$. With this presentation, he was transferred to our intensive care unit (ICU) for advanced life support (Figure 1).

Abbreviations: ALT, alanine aminotransferase; AKI, acute kidney injury; APACHE II, Physiologic Assessment and Chronic Health Evaluation II; AST, aspartate aminotransferase; RBC, red blood cell; RTC, renal tubular cells; CBA, cytometric bead array; COVID-19, coronavirus disease; CRP, C-reactive protein; CRRT, continuous renal replacement therapy; CRS, cytokine release syndrome; GFR, glomerular filtration rate; ICU, intensive care unit; IL, interleukin; RCA, regional citrate anticoagulation; RT-PCR, real-time polymerase chain reaction; SARS-CoV-2, severe acute respiratory syndrome coronavirus 2; SOFA, sequential organ failure assessment; VIE, Vasoactive-Inotropic Equivalent; VV-ECMO, venovenous extracorporeal membrane oxygenation.

\section{DISEASE ASSESSMENT}

\section{Clinical Parameters and SARS-CoV-2 Detection}

Acute disease severity was assessed using Acute Physiologic Assessment and Chronic Health Evaluation (APACHE) II scores collected on ICU admission and the next day of ICU stay (10). Sequential organ failure assessment (SOFA) scores were collected daily (11). Vasoactive-Inotropic Equivalent (VIE) scores were calculated from the infusion rates of vasopressors and inotropes and automatically summed up in the computerized database (12). Acute kidney injury was defined using the Kidney Disease: Improving Global Outcomes 2012 criteria (13). We also calculated the kinetic glomerular filtration rate (GFR), which forecasts future renal dysfunction derived from two consecutive days' serum creatinine data (14). Collection and detection of SARS-CoV-2 from biological samples were compliant with the World Health Organization guidance (15). Sputum, nasopharyngeal swabs, and oropharyngeal swabs were obtained for real-time PCR (RT-PCR). We determined the SARS$\mathrm{CoV}-2$ viral loads in each biological sample.

\section{Continuous Renal Replacement Therapy}

The indication for continuous renal replacement therapy (CRRT) was oliguria for more than $12 \mathrm{~h}$, with clinical evidence of fluid overload and hemodynamic compromise. We used a standard CRRT machine (Plasmaflex ${ }^{\circledR}$, Baxter ${ }^{\circledR}$, France) with either a standard AN69 based hemofilter (M150, Baxter ${ }^{\circledR}$, France) or a specialized hemofilter composed of AN69 and polyethyleneimine (AN69ST/PEI, oXiris ${ }^{\circledR}$, Baxter ${ }^{\circledR}$ France) used for increased clearance of cytokines and endotoxins in patients with sepsis (16). The CRRT prescription was compatible with the current standard (13). We used continuous venovenous hemodiafiltration (CVVHDF) with a dialysate flow of $10-15 \mathrm{~mL} / \mathrm{kg} / \mathrm{h}$ along with a filtration flow of $15-20 \mathrm{~mL} / \mathrm{kg} / \mathrm{h}$ via a 14 Fr-uncuffed tunneled catheter. To decrease filtration fraction and prolong circuit lives, we chose CVVHDF as the main treatment modality. The filtration fraction was set to below $20 \%$ to avoid pre-mature dysfunction of the circuit. The AN69ST/PEI hemofilter with increased adsorptive capacity for cytokines and endotoxins has been emergently approved by the United States in response to the COVID-19 pandemic under EUA200164 and has been granted permission for use in Taiwan in recent years. We set up CRRT with an AN69ST/PEI along with heparin or regional citrate anticoagulation (RCA) (17). We targeted the activated prothrombin time around 50-70s while using heparin and targeted a post-filter ionized calcium around 0.3-0.45 $\mathrm{mmol} / \mathrm{L}$ while using RCA (18).

\section{Veno-Venous Extracorporeal Membrane Oxygenation Life Support}

The indication for veno-venous extracorporeal membrane oxygenation (VV-ECMO) support was refractory hypoxemia. We cannulated the patient via the right internal jugular and femoral vein using the cut-down method. The VV-ECMO comprised a circuit with heparin-bound surfaces, an oxygenator (Affinity NT, Medtronic), a centrifugal pump (BPX-80 Bio-Pump Plus, 


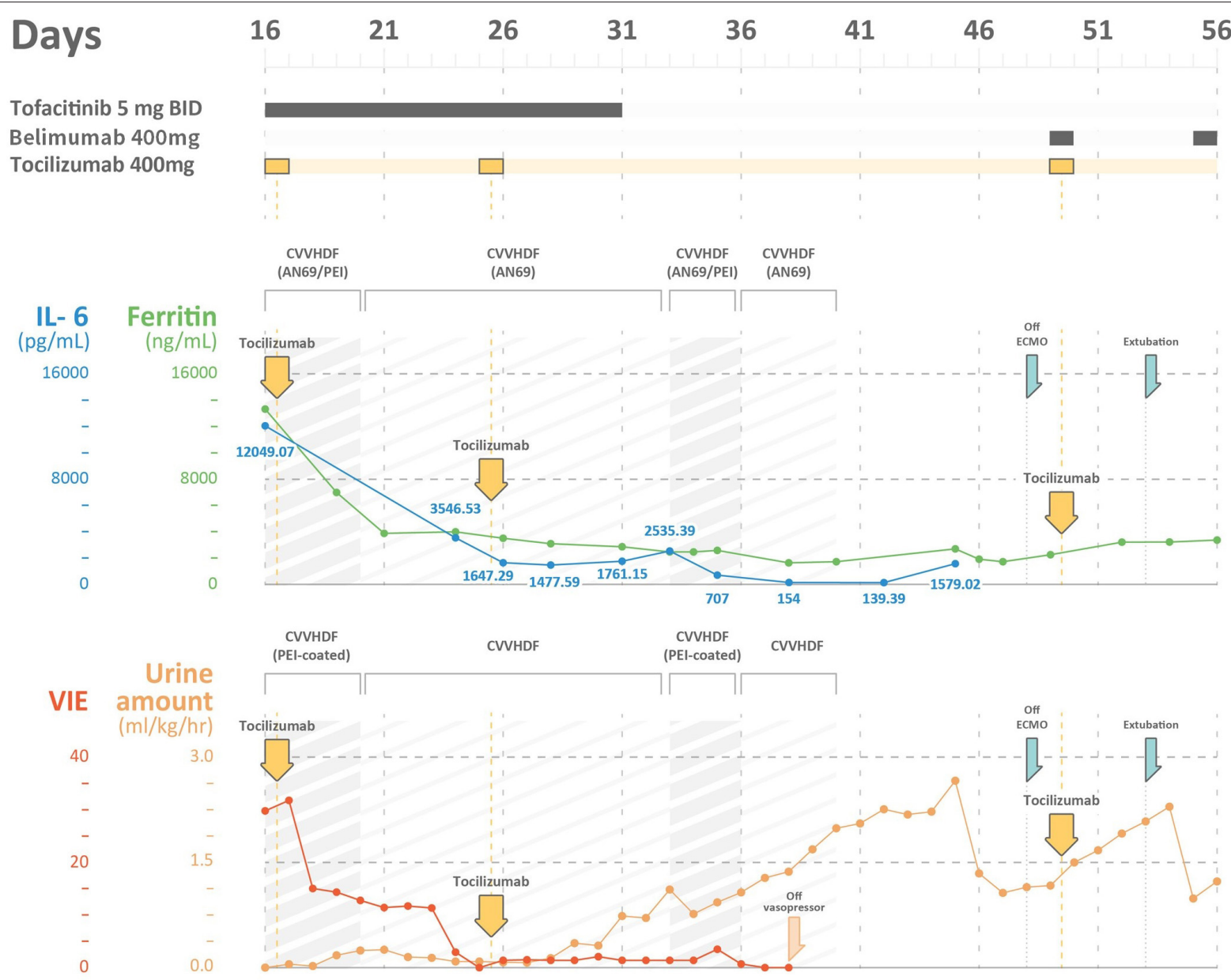

FIGURE 1 | The time course of ferritin levels, IL-6 levels, vasoactive-inotropic equivalent (VIE) scores, and urine amount in a severe COVID-19 patient with multi-organ failures. IL-6, interleukin 6; VIE, vasoactive-inotropic equivalent; CVVHDF, continuous venovenous hemodiafiltration; AN69ST/PEI: hemofilter with AN69 and polyethyleneimine.

Medtronic, Anaheim, CA, USA), and an oxygen-air blender (Model 3500 CP-G gas mixer, Sechrist, Anaheim, CA, USA) (19).

\section{Cytokine Measurement}

We used the cytometric bead array (CBA) method to determine cytokine levels (20). Approximately $5 \mathrm{~mL}$ peripheral blood samples were obtained from patients at each time point. Plasma was isolated after centrifugation at $1,500 \mathrm{rpm}$ for $10 \mathrm{~min}$. The concentrations of cytokines (interleukin [IL]-2, IL-4, IL-6, IL-10, IL-17, interferon- $\gamma$, and tumor necrosis factor in plasma were determined using cytometric bead array (CBA; BD Biosciences), according to the manufacturer's protocol. Briefly, $350 \mu \mathrm{L}$ samples were subjected to analysis in duplicate using the CBA kit on $\mathrm{BD}$ caliber cytometry. The concentrations of cytokines in culture supernatants were quantified using FCAP Array software v3.0 (20). We determined cytokine levels in samples collected from plasma and CRRT effluent. Plasma cytokine levels were checked at three-time points (before CRRT, hour 12, and 24) to determine the number of total cytokines removed using the area under the curve method. Because it was impossible to check the adsorptive capacity of AN69ST/PEI hemofilter, we checked cytokine levels from the CRRT effluent collected in the first $24 \mathrm{~h}$ on CRRT with the AN69ST/PEI hemofilter. The differences between these two values could approximate the number of cytokines absorbed by the hemofilter.

\section{Medications for Immune Modulation}

The patient was prescribed tocilizumab, tofacitinib, hydroxychloroquine, and belimumab during his hospital course. Tocilizumab acts as an IL-6 receptor blockade and has been approved for rheumatoid arthritis, giant cell arteritis, and chimeric antigen receptor (CAR) T cell-induced severe or life-threatening cytokine release syndrome (21). Tofacitinib is an inhibitor of Janus kinase (JAKs) 1 and 3. It also has partial selectivity to JAK 2 . Tofacitinib may suppress pro-inflammatory signaling including IL-6. It has not been tested in COVID-19 patients for cytokine blockade (22). Hydroxychloroquine is an anti-malarial drug and in vitro study confirmed its ability to 
inhibit SARS-CoV-2 (23). Antiphospholipid activities may be detected in COVID-19 patients. The mechanism of belimumab is to inhibit the binding of soluble circulating B lymphocyte stimulator to surface ligands of B cells, which may downregulate the anti-phospholipid activities (24).

\section{RESULTS}

Upon arrival to the ICU, the patient experienced rapid deterioration of oxygenation and hemodynamics. The body temperature was high $\left(38.3^{\circ} \mathrm{C}\right)$, arterial blood pressure was low $(79 / 51 \mathrm{mmHg})$, and he had tachycardia (heart rate, $136 \mathrm{bpm})$. He needed vasopressors with a VIE of 29.80: norepinephrine at 0.26 $\mathrm{mcg} / \mathrm{kg} / \mathrm{min}$, and dopamine at $3.7 \mu \mathrm{g} / \mathrm{kg} / \mathrm{min}$ were administered to maintain adequate mean artery pressure. The APACHE II score was 40 (corresponding to an estimated mortality rate of $85 \%$ ), and the SOFA score was 17 . There was a severe acute kidney injury (AKI) with an absence of urine output for more than $12 \mathrm{~h}$ and an increase in creatinine level to $3.1 \mathrm{mg} / \mathrm{dL}$. The kinetic GFR was calculated to be $0 \mathrm{~mL} / \mathrm{min}$ (14). Urinalysis showed dysmorphic red blood cells (RBCs) and renal tubular cells (RTCs), which indicated glomerulonephritis and acute tubular necrosis. A VV-ECMO circuit was promptly set for refractory hypoxemia (2). His laboratory values indicated a relative lymphopenia (lymphocyte percentage: $6.1 \%$, with an absolute count of $722 / \mu \mathrm{L}$ ). The ferritin level increased to $13,317 \mathrm{ng} / \mathrm{mL}$, and the triglyceride level was $446 \mathrm{mg} / \mathrm{dL}$. The AST level was $121 \mathrm{U} / \mathrm{L}$, along with an ALT level of $47 \mathrm{U} / \mathrm{L}$. The cytokine profiles displayed an extremely high IL-6 level (12,049.07 pg/mL). A rapidly evolving CRS was likely. In addition, with rapidly elevating procalcitonin levels (from 0.15 to $>100 \mathrm{pg} / \mathrm{mL}$ ), ceftazidime and levofloxacin were prescribed empirically for concern of a superimposed bacterial infection, although the bacterial cultures did not yield until discharge. CRRT with an AN69ST/PEI hemofilter was delivered using a separate uncuffed tunneled catheter. Intravenous immunoglobulin (1 $\mathrm{g} / \mathrm{kg} /$ day) was administered for two consecutive days for hypogammaglobulinemia (immunoglobulin G: $629 \mathrm{mg} / \mathrm{dL}$ ).

The poor renal reserve precluded the patient from remdesivir therapy. Hydroxychloroquine with $400 \mathrm{mg}$ twice daily was continued, and tocilizumab with a total dose of $400 \mathrm{mg}(5.2 \mathrm{mg} / \mathrm{kg})$ was prescribed $2 \mathrm{~h}$ after the initiation of CRRT. Tofacitinib (5 mg twice daily) was also prescribed for suspected antiphospholipid activity. We changed the AN69ST/PEA hemofilter circuit every $24 \mathrm{~h}$ for better clearance of cytokine/endotoxin. After 26-h treatment, the patient's hemodynamics improved. On day 20, we changed the CRRT hemofilter to an AN69 based hemofilter, after 3 days of cytokine adsorptive treatment. The viral load from the sputum was 2,762 copies/mL. Dopamine and norepinephrine were tapered off on day 22 (after 6-day cytokine-targeted therapy). The ferritin level declined from $13,317 \mathrm{ng} / \mathrm{mL}$ on day 16 to $3,875 \mathrm{ng} / \mathrm{mL}$ on day 21 . The total bilirubin level was $2.28 \mathrm{mg} / \mathrm{dL}$, and the triglyceride level decreased to $320 \mathrm{mg} / \mathrm{dL}$. Another dose of tocilizumab with the same dosage of $400 \mathrm{mg}$ was administered on day 25. The viral load at that time from sputum was 1.35 million copies $/ \mathrm{mL}$ (Table 1).

On day 33, he was found to have an elevation of ferritin level and persistent tachycardia. Low dose norepinephrine (0.02 $\mathrm{mcg} / \mathrm{kg} / \mathrm{min}$ ) was added. Ferritin levels increased from 1,761.15 to $2,535.39 \mathrm{ng} / \mathrm{mL}$, and IL-6 levels increased from $1,477.59$ to $2,535.39 \mathrm{pg} / \mathrm{mL}$. There was persistent viral shedding from sputum with a viral load of 99.65 copies $/ \mathrm{mL}$. For suspected recurrence of CRS, another session of CRRT with an AN69ST/PEA hemofilter was performed on day 34, and the IL- 6 level decreased to 707 $\mathrm{pg} / \mathrm{mL}$ on day 35 and $154 \mathrm{pg} / \mathrm{mL}$ on day 38 . We collected the CRRT effluent to estimate the removal of IL- 6 from either CRRT or hemadsorption. The pre-AN69ST/PEI IL-6 level was 715.85 $\mathrm{pg} / \mathrm{mL}$, and the level $24 \mathrm{~h}$ later was $541.59 \mathrm{pg} / \mathrm{mL}$. A total of $2,369,936 \mathrm{pg}$ of IL-6 was removed, with $483,600 \mathrm{pg}(20.4 \%)$ removed through the CRRT effluent and 1,886,336 pg (79.6\%) removed by hemadsorptions. With adequate urine output and decreased oxygen demand, CRRT was discontinued on day 40.

He developed another recurrence of CRS on day 45 with an elevation of IL-6 (1,579 pg/mL) and ferritin $(2,690 \mathrm{ng} / \mathrm{mL})$ levels. An additional dose of tocilizumab $400 \mathrm{mg}$ and belimumab (B-cell activating factor inhibitor) was administered. ECMO was terminated on day 49 , and he was successfully extubated on day 52 (Figures 2B,C). The urine output was 2,000-2,500 $\mathrm{mL} /$ day after extubation. The urinalysis findings of dysmorphic $\mathrm{RBC}$, glycosuria, leukocyturia, and RTC resolved. The followup diluted Russell viper venom test for lupus anticoagulant level was negative. The sputum RT-PCR revealed persistently positive results for SARS-CoV-2 until day 52. The patient was discharged without oxygen support on day 70 (Figure 1).

\section{DISCUSSION}

Critically ill COVID-19 patients, especially those with multiorgan failure, such as cardiovascular collapse, AKI, CRS, or thromboembolic events, challenge clinicians with a substantial risk of patient mortality (25-27) Thus, treatment for these patients should target multiple derangements induced by COVID-19, including ventilator support and ECMO for refractory hypoxemia, CRRT for AKI and extracorporeal cytokine removal, and biologic agents for cytokine blockade. We used a lung-protective strategy in mechanical ventilation and a CRRT with an AN69ST/PEA hemofilter for uremic toxin removal, volume management, cytokine, and endotoxin removal. VV-ECMO was initiated for oxygenation support. In addition, immunomodulating agents were prescribed to control CRS. We successfully treated a critically ill COVID-19 patient with predicted high mortality. This report also demonstrates a successful experience for managing severe COVID-19 without effective antiviral agents. We also recommend timely implementation of the cytokine-targeted strategy combining CRRT with an AN69ST/PEI hemofilter and tocilizumab for severe COVID-19 with CRS and multi-organ failure.

CRS in COVID-19 originates from a dysregulated immune response to SARS-CoV-2, which is not new to coronary virus infection $(6,28)$. Treatment for CRS includes removal of the 
TABLE 1 | Baseline characteristics, treatment courses, and relevant data of the reported patient.

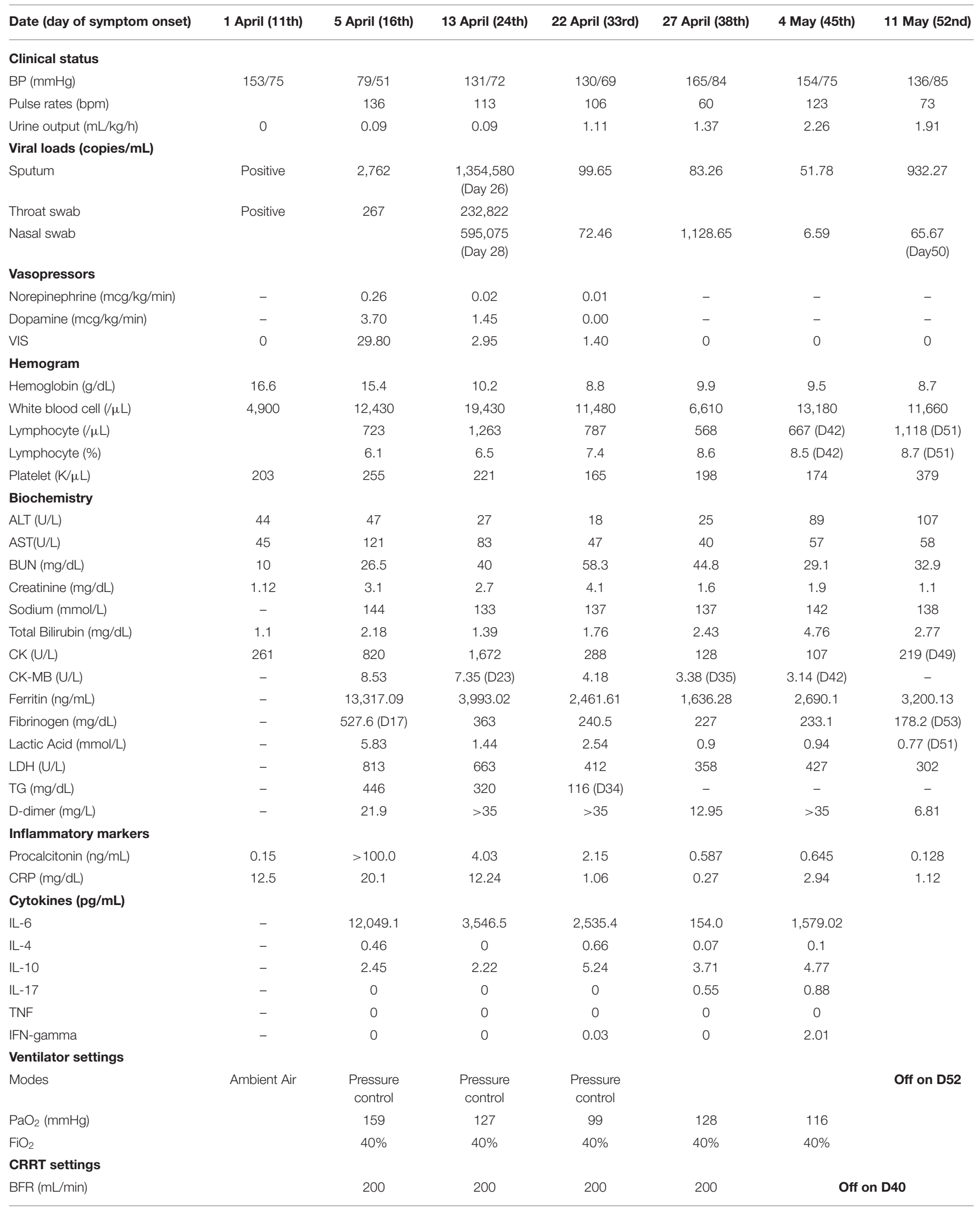


TABLE 1 | Continued

\begin{tabular}{|c|c|c|c|c|c|c|c|}
\hline Date (day of symptom onset) & 1 April (11th) & 5 April (16th) & 13 April (24th) & 22 April (33rd) & 27 April (38th) & 4 May (45th) & 11 May (52nd) \\
\hline Total wastes (mL/h) & & 2,000 & 2,000 & 2,000 & 1,700 & & \\
\hline Anticoagulant & & $\mathrm{RCA}$ & Heparin & Heparin & Heparin & & \\
\hline \multicolumn{8}{|l|}{ VV-ECMO Settings } \\
\hline $\mathrm{BFR}(\mathrm{L} / \mathrm{min})$ & & 3.7 & 3.75 & 3.3 & 3.91 & 2.58 & Off on D49 \\
\hline $\mathrm{ECMO} \mathrm{FiO}_{2}$ & & $100 \%$ & $50 \%$ & $100 \%$ & $40 \%$ & $21 \%$ & \\
\hline \multicolumn{8}{|l|}{ Medications } \\
\hline Methylprednisolone (mg/kg/day) & & - & - & 0.4 & 0.4 & 0.3 & 0.4 \\
\hline Tocilizumab 400 mg & & & $\checkmark(\mathrm{D} 25)$ & & & $\checkmark(\mathrm{D} 49)$ & \\
\hline Tofacitinib 5 mg BID & & $\checkmark$ & $\checkmark$ & & & & \\
\hline Belimumab 400 mg & & & & & & $\checkmark(\mathrm{D} 49)$ & \\
\hline AN69ST/PEI & & $\checkmark$ & & $\checkmark$ & & & \\
\hline
\end{tabular}

COVID-19, coronavirus disease; ICU, intensive care unit; MV, mechanical ventilation; ECMO, extracorporeal membrane oxygenation; CRRT, continuous renal replacement therapy; AN69ST/PEI, hemofilter with AN69 and polyethyleneimine; BP, arterial blood pressure; VIS, vasoactive inotropic score; ALT, alanine aminotransferase; AST, aspartate aminotransferase; $C K$, creatine kinase; $L D H$, lactate dehydrogenase; BUN, blood urea nitrogen; $C K$, creatinine kinase; $C K-M B$, creatinine kinase muscle and brain form; CRP; TG, triglyceride; $C$-reactive protein; IL, interleukin; TNF, tumor necrotizing factor; IFN, interferon; $\mathrm{FiO}_{2}$, fraction of inhaled oxygen; W-ECMO, veno-venous extracorporeal membrane oxygenation; BPF, blood flow rates.

$\checkmark$ : usage of this device or medications.
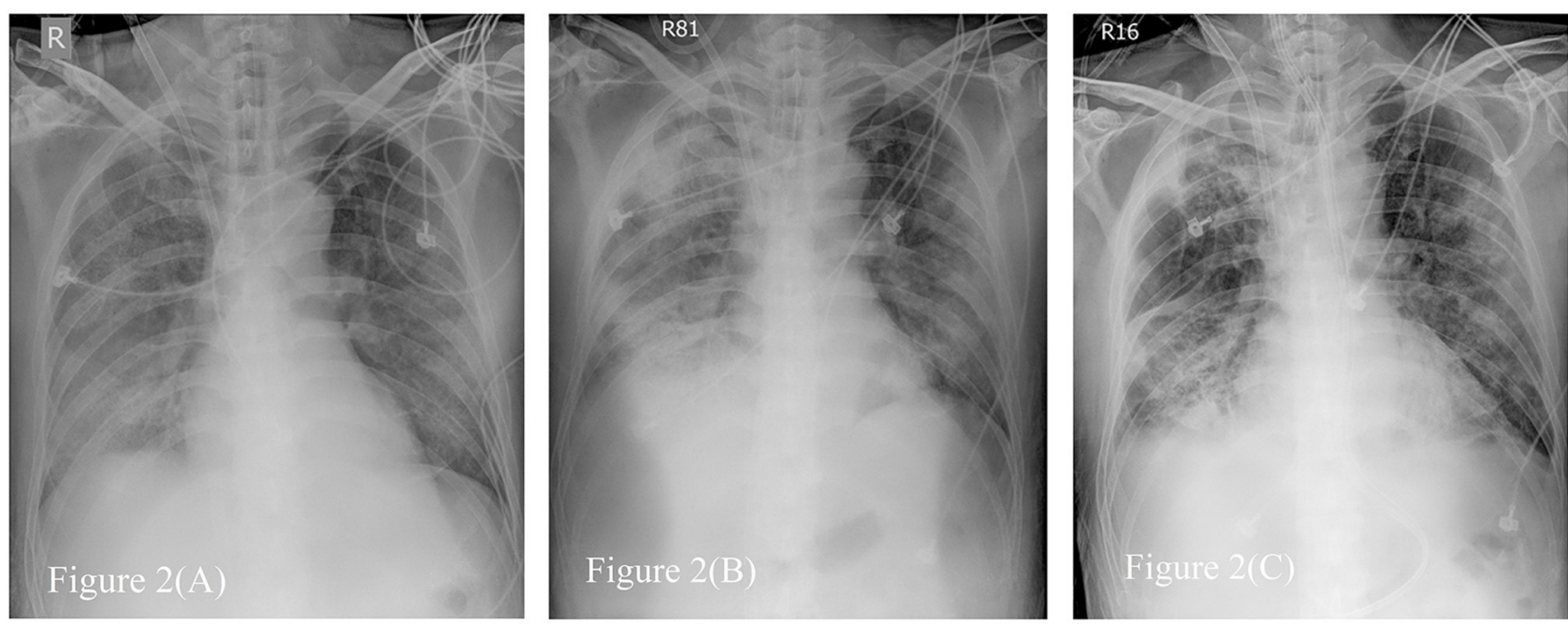

FIGURE 2 | Serial Chest radiographs of the patient. (A) The film showing diffuse alveolar process over bilateral lung field, especially right lower lung field just after extracorporeal membrane oxygenator (ECMO setup). (B) The film revealing consolidation over both lungs when first time we tried to wean from ECMO. (C) The film on the day before removing $\mathrm{ECMO}$, demonstrating fibrotic change as lung filed in (B).

offending pathogen and limiting the propagation of cytokines, in order to limit organ damage. Effective anti-SARS-CoV2 therapies are not available at this time (29). Mechanical removal of the offending cytokines was reasonable. Methods for cytokine removal include direct hemoperfusion, conventional CRRT, high-dose CRRT, or CRRT with hemofilters with higher cut-off membranes (5). In patients with severe sepsis, CRRT with an AN69ST/PEA hemofilter might be associated with better outcomes. However, the only randomized control trial failed to balance its intervention group and placebo group and showed no benefit (16). However, CRRT with an AN69ST/PEA hemofilter has been shown to have better cytokine removal properties than conventional CRRT (16). This was also evident in our observation.
In addition to cytokine removal, we also used tocilizumab to neutralize the deleterious effects of IL-6. Recent publications have suggested that tocilizumab might mitigate CRS, although the studies were limited by non-controlled designs or case reports $(30,31)$ In their reports, the IL-6 level before tocilizumab treatment was $41 \mathrm{ng} / \mathrm{L}$ (interquartile range [IQR]: 10-102 ng/L), and elevated to $1,812 \mathrm{ng} / \mathrm{L}$ (IQR: $375-2,600 \mathrm{ng} / \mathrm{L}$ ). Our case had a much higher pre-treatment level $(12,049.07 \mathrm{ng} / \mathrm{L})$; further, concurrent CRRT with an AN69ST/PEA hemofilter, the level rapidly reduced to $3,546.53 \mathrm{ng} / \mathrm{L}$, and the second course of CRRT with an AN69ST/PEA hemofilter further reduced the IL-6 level from 2,535.39 ng/L to $139.39 \mathrm{ng} / \mathrm{L}$. A retrospective study, based on data from 85 patients, demonstrated lower mortality rates. However, no IL-6 data were available in this 
study (32). We propose that there are synergistic benefits from combined CRRT with AN69ST/PEA hemofilter therapy and tocilizumab therapy.

Another concern about immunomodulation therapy in the absence of effective antiviral therapy is the potential for prolonged viral infection. In our patient, the viral shedding persisted for over 60 days. There is an urgent, but unmet, need for effective antiviral therapy in patients with severe COVID-19 and renal dysfunction. Remdesivir was not be considered universally to be an effective anti-viral agent for SARS-CoV-2; there were negative clinical trials, although the analytic methods may have led to negative trials (33). Of note, remdesivir is not indicated for patients with renal dysfunction $\left(<30 \mathrm{~mL} / 1.73 \mathrm{~m}^{2}\right)(4)$.

VV-ECMO is a reasonable option for refractory ARDS caused by viral pneumonia. Based on the recent meta-analysis, the 90day mortality rates decreased in the VV-ECMO arm (relative risk $[\mathrm{RR}]=0.73$ [95\% CI 0.58-0.92]; $p=0.008$ ) (34). But bleeding risk was one major complication for ECMO. The current guideline still use VV-ECMO as salvage therapy for severe ARDS with refractory hypoxemia. Advanced age, morbid obesity, and immunocompromised status are relative contra-indications as stated by ESLO guideline. It is not clear whether ECMO would be beneficial in patients with severe ARDS. But good organ support and meticulous prevention and management are keys to success in our patient (35). AKI is a common complication in patients with severe COVID-19 and is associated with worse outcomes (26). Interestingly, renal complications are not limited to AKI; hematuria, proteinuria, or glycosuria also develop with COVID-19 (9). Evidence has shown that hematuria, proteinuria, and glycosuria develop during the critical illness and subside after renal recovery, similar to that observed in our patient (36). We had limited renal biopsy data to explore the underlying pathogenesis of SARS-CoV-2 infections (37), although data from SARS-CoV may give us hints that renal damage from COVID-19 is diverse (38). The increased hematuria and proteinuria are also associated with adverse outcomes (36).

The plain radiograph is less sensitive than chest CT, but chest radiography is typically the first-line imaging modality ordered for patients with suspected COVID-19. Compared to typical bacteremic pneumonia, the correlation of chest radiography to oxygen demand is poor. In our patient, more consolidation was detected on chest radiography when less $\mathrm{O}_{2}$ demand, shown in Figure 2. The follow-up chest radiograph revealed sequelae of pulmonary fibrosis. It is not useful to predict severity by image evaluation once before diagnosis or during treatment for COVID-19 (39).

There were some limitations to this case report. As a report of a single case, it is not possible to generalize the experience to the whole patient group with severe COVID-19. However, we are confident that this report would inspire future clinical trials involving the cytokine-targeted therapy, proposed in this

\section{REFERENCES}

1. Zavascki AP, Falci DR. Clinical characteristics of Covid-19 in China. N Engl J Med. (2020) 382:1859. doi: 10.1056/NEJMc2005203 study. Second, the patient was treated in an area where there were few critical cases and thus, cannot be generalized to a medical system overwhelmed by the COVID-19 pandemic (2). However, if further clinical trials prove its efficacy, timely cytokine-targeted therapy for critically ill patients with COVID-19 is likely to improve patient outcomes. Third, we did not use antiviral therapy for this patient. Poor renal reserve precluded the patient from remdesivir therapy $(4,29)$.

In summary, we demonstrated in this report that timely combined hemoperfusion with cytokine adsorptive capacity and anti-cytokine therapy may successfully treat COVID-19 patients with devastating CRS. For the rapid progression of severe COVID-19 ARDS, cytokine releasing syndrome, acute kidney injury, and multiple organ failure. We would suggest timely initiation of life support and use of multimodality treatment for blockade of cytokine effects (by tocilizumab) and rapid removal of pro-inflammatory cytokines to limit organ damage. This further highlights the importance of implementing cytokine-targeted therapy in severe COVID-19 guided by precise measurement of disease activity.

\section{DATA AVAILABILITY STATEMENT}

The raw data supporting the conclusions of this article will be made available by the authors, without undue reservation.

\section{ETHICS STATEMENT}

Written informed consent was obtained from the individual(s) for the publication of any potentially identifiable images or data included in this article.

\section{AUTHOR CONTRIBUTIONS}

TH, Y-CC, C-HW, S-YC, B-LC, and S-CH performed the data collection. TH, Y-CC, C-HW, Y-CY, S-CK, and AT wrote the manuscript. J-TW, C-JY, and S-CC carried out the project administration. All authors contributed to the article and approved the submitted version.

\section{FUNDING}

This study was funded by the National Taiwan University Hospital (NTUH-109-P13), the Mrs. Hsiu-Chin Lee Kidney Research Fund, and the Taiwan Health Foundation.

\section{SUPPLEMENTARY MATERIAL}

The Supplementary Material for this article can be found online at: https://www.frontiersin.org/articles/10.3389/fmed. 2021.649583/full\#supplementary-material

2. Zhao XY, Xu XX, Yin HS, Hu QM, Xiong T, Tang YY, et al. Clinical characteristics of patients with 2019 coronavirus disease in a non-Wuhan area of Hubei Province, China: a retrospective study. BMC Infect Dis. (2020) 20:311. doi: 10.1186/s12879-020-05010-w 
3. Chen T, Wu D, Chen H, Yan W, Yang D, Chen G, et al. Clinical characteristics of 113 deceased patients with coronavirus disease 2019: retrospective study. BMJ. (2020) 368:m1091. doi: 10.1136/bmj.m1091

4. Grein J, Ohmagari N, Shin D, Diaz G, Asperges E, Castagna A, et al. Compassionate use of remdesivir for patients with severe Covid-19. N Engl J Med. (2020) 382:2327-36. doi: 10.1056/NEJMoa2007016

5. Ronco C, Reis T. Kidney involvement in COVID-19 and rationale for extracorporeal therapies. Nat Rev Nephrol. (2020) 16:308-10. doi: 10.1038/s41581-020-0284-7

6. Moore JB, June CH. Cytokine release syndrome in severe COVID-19. Science. (2020) 368:473-4. doi: 10.1126/science.abb8925

7. McGonagle D, Sharif K, O'Regan A, Bridgewood C. The role of cytokines including interleukin-6 in COVID-19 induced pneumonia and macrophage activation syndrome-like disease. Autoimmun Rev. (2020) 19:102537. doi: 10.1016/j.autrev.2020.102537

8. Connell NT, Battinelli EM, Connors JM. Coagulopathy of COVID-19 and antiphospholipid antibodies. J Thromb Haemost. (2020). doi: 10.1111/jth.14893

9. Batlle D, Soler MJ, Sparks MA, Hiremath S, South AM, Welling PA, et al. Acute kidney injury in COVID-19: emerging evidence of a distinct pathophysiology. J Am Soc Nephrol. (2020) 31:1380-3. doi: 10.1681/ASN. 2020040419

10. Knaus WA, Draper EA, Wagner DP, Zimmerman JE. APACHE II: a severity of disease classification system. Crit Care Med. (1985) 13:81829. doi: 10.1097/00003246-198510000-00009

11. Vincent JL, Moreno R, Takala J, Willatts S, De Mendonca A, Bruining H, et al. The SOFA (Sepsis-related Organ Failure Assessment) score to describe organ dysfunction/failure. On behalf of the Working Group on Sepsis-Related Problems of the European Society of Intensive Care Medicine. Intensive Care Med. (1996) 22:707-10. doi: 10.1007/BF01709751

12. Chen YS, Yu HY, Huang SC, Chiu KM, Lin TY, Lai LP, et al. Experience and result of extracorporeal membrane oxygenation in treating fulminant myocarditis with shock: what mechanical support should be considered first? J Heart Lung Transplant. (2005) 24:81-7. doi: 10.1016/j.healun.2003.09.038

13. Tolwani A. Continuous renal-replacement therapy for acute kidney injury. $N$ Engl J Med. (2012) 367:2505-14. doi: 10.1056/NEJMct1206045

14. Chen S. Retooling the creatinine clearance equation to estimate kinetic GFR when the plasma creatinine is changing acutely. J Am Soc Nephrol. (2013) 24:877-88. doi: 10.1681/ASN.2012070653

15. World Health Organization. Laboratory Testing for Coronavirus Disease (COVID-19) in Suspected Human Cases (2020).

16. Broman ME, Hansson F, Vincent JL, Bodelsson M. Endotoxin and cytokine reducing properties of the oXiris membrane in patients with septic shock: a randomized crossover double-blind study. PLoS One. (2019) 14:e220444. doi: 10.1371/journal.pone.0220444

17. Rimmele T, Kellum JA. Clinical review: blood purification for sepsis. Crit Care. (2011) 15:205. doi: 10.1186/cc9411

18. Schneider AG, Journois D, Rimmele T. Complications of regional citrate anticoagulation: accumulation or overload? Crit Care. (2017) 21:281. doi: 10.1186/s13054-017-1880-1

19. Chou NK, Chen YS, Ko WJ, Huang SC, Chao A, Jan GJ, et al. Application of extracorporeal membrane oxygenation in adult burn patients. Artif Organs. (2001) 25:622-6. doi: 10.1046/j.1525-1594.2001.025008622.x

20. Shu CC, Wang JY, Wu MF, Lai HC, Chiang BL, Yu CJ. Interleukin 23/interleukin 17 axis activated by Mycobacterium avium complex (MAC) is attenuated in patients with MAC-lung disease. Tuberculosis (Edinb). (2018) 110:7-14. doi: 10.1016/j.tube.2018.03.001

21. Fitzgerald JC, Weiss SL, Maude SL, Barrett DM, Lacey SF, Melenhorst JJ, et al. Cytokine release syndrome after chimeric antigen receptor $\mathrm{T}$ cell therapy for acute lymphoblastic leukemia. Crit Care Med. (2017) 45:e124e31. doi: 10.1097/CCM.0000000000002053

22. Migita K, Izumi Y, Jiuchi Y, Kozuru H, Kawahara C, Izumi M, et al. Effects of Janus kinase inhibitor tofacitinib on circulating serum amyloid A and interleukin-6 during treatment for rheumatoid arthritis. Clin Exp Immunol. (2014) 175:208-14. doi: 10.1111/cei.12234

23. Yao X, Ye F, Zhang M, Cui C, Huang B, Niu P, et al. In vitro antiviral activity and projection of optimized dosing design of hydroxychloroquine for the treatment of severe acute respiratory syndrome coronavirus 2 (SARS-CoV-2). Clin Infect Dis. (2020) 71:732-9. doi: 10.1093/cid/ciaa237
24. Chatzidionysiou K, Samoli E, Sfikakis PP, Tektonidou MG. Effect of belimumab treatment on antiphospholipid antibody levels: post-hoc analysis based on two randomised placebo-controlled trials in systemic lupus erythematosus. Ann Rheum Dis. (2020) 79:304-7. doi: 10.1136/annrheumdis-2019-216367

25. Shi S, Qin M, Cai Y, Liu T, Shen B, Yang F, et al. Characteristics and clinical significance of myocardial injury in patients with severe coronavirus disease 2019. Eur Heart J. (2020) 41:2070-9. doi: 10.1093/eurheartj/ehaa408

26. Yang $\mathrm{X}, \mathrm{Yu} \mathrm{Y}, \mathrm{Xu}$ J, Shu H, Xia J, Liu H, et al. Clinical course and outcomes of critically ill patients with SARS-CoV-2 pneumonia in Wuhan, China: a single-centered, retrospective, observational study. Lancet Respir Med. (2020) 8:475-81. doi: 10.1016/S2213-2600(20)30079-5

27. Connors JM, Levy JH. COVID-19 and its implications for thrombosis and anticoagulation. Blood. (2020) 135:2033-40. doi: 10.1182/blood.2020006000

28. Channappanavar R, Perlman S. Pathogenic human coronavirus infections: causes and consequences of cytokine storm and immunopathology. Semin Immunopathol. (2017) 39:529-39. doi: 10.1007/s00281-017-0629-x

29. Cao B, Wang Y, Wen D, Liu W, Wang J, Fan G, et al. A trial of lopinavirritonavir in adults hospitalized with severe Covid-19. N Engl J Med. (2020) 382:1787-99. doi: 10.1056/NEJMoa2001282

30. Radbel J, Narayanan N, Bhatt PJ. Use of tocilizumab for COVID-19-induced cytokine release syndrome: a cautionary case report. Chest. (2020) 158:e15e9. doi: 10.1016/j.chest.2020.04.024

31. Toniati P, Piva S, Cattalini M, Garrafa E, Regola F, Castelli F, et al. Tocilizumab for the treatment of severe COVID-19 pneumonia with hyperinflammatory syndrome and acute respiratory failure: a single center study of 100 patients in Brescia, Italy. Autoimmun Rev. (2020) 19:102568. doi: 10.1016/j.autrev.2020.102568

32. Capra R, De Rossi N, Mattioli F, Romanelli G, Scarpazza C, Sormani MP, et al. Impact of low dose tocilizumab on mortality rate in patients with COVID-19 related pneumonia. Eur J Intern Med. (2020) 76:315. doi: 10.1016/j.ejim.2020.05.009

33. McCaw ZR, Tian L, Vassy JL, Ritchie CS, Lee CC, Kim DH, et al. How to quantify and interpret treatment effects in comparative clinical studies of COVID-19. Ann Intern Med. (2020) 173:632-7.. doi: 10.7326/M20-4044

34. Munshi L, Walkey A, Goligher E, Pham T, Uleryk EM, Fan E. Venovenous extracorporeal membrane oxygenation for acute respiratory distress syndrome: a systematic review and meta-analysis. Lancet Respir Med. (2019) 7:163-72. doi: 10.1016/S2213-2600(18)30452-1

35. Shekar K, Badulak J, Peek G, Boeken U, Dalton HJ, Arora L, et al Extracorporeal Life Support Organization COVID-19 Interim Guidelines: a consensus document from an international group of interdisciplinary extracorporeal membrane oxygenation providers. ASAIO J. (2020) 66:70721. doi: 10.1097/MAT.0000000000001193

36. Pei G, Zhang Z, Peng J, Liu L, Zhang C, Yu C, et al. Renal involvement and early prognosis in patients with COVID-19 pneumonia. J Am Soc Nephrol. (2020) 31:1157-65. doi: 10.1681/ASN.2020030276

37. Su H, Yang M, Wan C, Yi LX, Tang F, Zhu HY, et al. Renal histopathological analysis of 26 postmortem findings of patients with COVID-19 in China. Kidney Int. (2020) 98:219-27. doi: 10.1016/j.kint.2020.04.003

38. Wu VC, Huang JW, Hsueh PR, Yang YF, Tsai HB, Kan WC, et al. Renal hypouricemia is an ominous sign in patients with severe acute respiratory syndrome. Am J Kidney Dis. (2005) 45:88-95. doi: 10.1053/j.ajkd.2004.09.031

39. Wong HYF, Lam HYS, Fong AH, Leung ST, Chin TW, Lo CSY, et al. Frequency and distribution of chest radiographic findings in patients positive for COVID-19. Radiology. (2020) 296:E72-E8. doi: 10.1148/radiol.20202 01160

Conflict of Interest: The authors declare that the research was conducted in the absence of any commercial or financial relationships that could be construed as a potential conflict of interest.

Copyright $\odot 2021$ Huang, Chien, Wang, Chang, Wang, Hsieh, Yeh, Ku, Yu, Chiang, Chang and Tolwani. This is an open-access article distributed under the terms of the Creative Commons Attribution License (CC BY). The use, distribution or reproduction in other forums is permitted, provided the original author(s) and the copyright owner(s) are credited and that the original publication in this journal is cited, in accordance with accepted academic practice. No use, distribution or reproduction is permitted which does not comply with these terms. 